Mens

revue d'histoire intellectuelle de l'Amérique française

MENS

\title{
Les Éditions Mille Roches (1976-1989) : une mission régionale
}

\section{Caroline Béland}

Volume 2, numéro 2, printemps 2002

URI : https://id.erudit.org/iderudit/1024610ar

DOI : https://doi.org/10.7202/1024610ar

Aller au sommaire du numéro

Éditeur(s)

Centre de recherche en civilisation canadienne-française

ISSN

1492-8647 (imprimé)

1927-9299 (numérique)

Découvrir la revue

Citer cet article

Béland, C. (2002). Les Éditions Mille Roches (1976-1989) : une mission régionale. Mens, 2(2), 233-259. https://doi.org/10.7202/1024610ar

\section{Résumé de l'article}

Le présent article fait connaître un éditeur situé dans le Haut-Richelieu qui a revêtu une grande importance pour sa région sur les plans culturel, historique et littéraire, les Éditions Mille Roches. De par son historique, son

fonctionnement ainsi que sa politique éditoriale, la maison d'édition a toujours privilégié les ressources régionales dans le but de faire connaître et progresser le Haut-Richelieu. Bénéficiant de bon nombre d'appuis locaux, la publication de plusieurs titres a permis de créer un patrimoine littéraire et culturel qui, jusque-là, était inexistant dans cette région. Pendant ses treize années d'existence, l'éditeur a su utiliser toutes les ressources de sa région afin de publier des titres de qualité qui étaient fort susceptibles d'intéresser sa population. D'un autre côté, de nombreux acteurs ont participé à la mission régionale de l'éditeur, bâtissant ainsi un sentiment d'appartenance qui ne s'est jamais démenti tout au long de l'existence de la maison. 


\section{LES ÉDITIONS MILLE ROCHES (1976-1989) : UNE MISSION RÉGIONALE}

Caroline Béland ${ }^{1}$

\section{RÉSUMÉ}

Le présent article fait connaître un éditeur situé dans le HautRichelieu qui a revêtu une grande importance pour sa région sur les plans culturel, historique et littéraire, les Éditions Mille Roches. De par son historique, son fonctionnement ainsi que sa politique éditoriale, la maison d'édition a toujours privilégié les ressources régionales dans le but de faire connaitre et progresser le Haut-Richelieu. Bénéficiant de bon nombre d'appuis locaux, la publication de plusieurs titres a permis de créer un patrimoine littéraire et culturel qui, jusque-là, était inexistant dans cette région. Pendant ses treize années d'existence, l'éditeur a su utiliser toutes les ressources de sa région afin de publier des titres de qualité qui étaient fort susceptibles d'intéresser sa population. D'un autre côté, de nombreux acteurs ont participé à la mission régionale de l'éditeur, bâtissant ainsi un sentiment d'appartenance qui ne s'est jamais démenti tout au long de l'existence de la maison.

\section{ABSTRACT}

This article traces the bistory of the Éditions Mille Roches, a publishing house whose cultural, historic and literary contribution to Quebec's Upper Richelieu Valley has been significant. In its dayto-day activities and its editorial policy, the firm always sought to publicize and develop the region's cultural resources. Indeed, with local support, Mille Roches published several titles that helped to create a regional literary canon and stimulated the Upper Richelieu 
Valley's cultural development. During its thirteen years of existence, the firm mobilized the region's cultural, literary and intellectual resources in an effort to publish volumes likely to interest the Valley's inbabitants. Moreover, Mille Roches' publishing ventures belped to develop a sense of regional identity and belonging in the Upper R $i$ chelieu Valley.

Même parmi la population qui réside dans le HautRichelieu depuis bon nombre d'années, peu de gens connaissent les Éditions Mille Roches. Cette maison d'édition, fondée en 1976 à Saint-Jean-sur-Richelieu (Montérégie), a pourtant été la première et la seule maison d'importance dans cette région. Il n'est pas étonnant qu'elle soit méconnue dans son propre patelin, puisque si l'on regarde de plus près le contexte historique entourant sa fondation, on remarque d'emblée que l'édition à vocation régionaliste n'en était alors qu'à ses balbutiements. Des éditeurs importants se sont installés à Montréal dans les deux premiers tiers du $\mathrm{XX}^{\mathrm{e}}$ (Beauchemin, Fides, l'Hexagone, Éditions de l'Homme, Leméac, pour ne citer que celles-là), mais très peu prennent le risque de s'établir en région. Avant les années soixante-dix, il existe peu d'activité éditoriale en dehors de Montréal. Fernand Harvey explique cette situation par le fait que

Les années 1940 et 1950 ont accentué la concentration de la fonction éditoriale à Montréal, et cette tendance a naturellement pris de l'ampleur à partir de la révolution tranquille. L'édition montréalaise devenait ainsi le prolongement naturel de l'institution littéraire en pleine expansion ${ }^{2}$.

Mis à part quelques éditeurs situés dans les villes de Québec, Trois-Rivières et Sherbrooke, aucune maison ne décide de défier la métropole. De plus, ces «éditeurs régio- 
naux ${ }^{3} »$ concentrent leurs activités éditoriales la plupart du temps autour d'une association régionale ou de la production d'un périodique auxquelles participent des écrivains de la région. Ils ne peuvent donc pas être qualifiés d'éditeurs puisque leur but premier n'est pas l'édition de livres.

La situation se modifie au cours des années soixante et soixante-dix puisqu'une dizaine d'éditeurs choisissent de s'établir en région, dont les plus connus, et encore actifs aujourd'hui, sont les Éditions du Noroît (Saint-Lambert) et les Écrits des Forges (Trois-Rivières), cette dernière maison ayant été fondée par le poète Gatien Lapointe. On remarque aussi un certain essor de l'édition en Montérégie puisque les Éditions du Broquet, les Éditions Héritage, ainsi que l'éditeur qui nous intéresse ici, les Éditions Mille Roches, y ont été fondées au cours de ces deux décennies. Les années soixante-dix voient également la naissance de l'édition franco-ontarienne avec la fondation, en 1973, des Éditions Prise de Parole (Sudbury), dont les assises et la vocation sont largement régionales.

Cependant, on ne peut se fier au seul critère géographique pour qualifier un éditeur de «régional». En effet, un éditeur peut s'installer en dehors de Montréal sans avoir le dessein de faire progresser sa région. Il y a donc une nette disctinction à faire entre «éditeur en région» et «éditeur régional». Le premier s'établit en dehors de Montréal pour faire ses activités éditoriales au même titre qu'un éditeur montréalais, sans souci particulier envers sa région, tandis que le second s'établit en région dans le but premier de faire connaître ses richesses culturelles, historiques et littéraires à sa population et de la faire progresser par ses publications. Mis à part le critère géographique, nous avons donc établi quatre critères qui définissent un éditeur régional. Tout d'abord, nous avons remarqué que plusieurs éditeurs 
situés en région ont été fondés par plus d'une personne, donc par une équipe qui a comme souci de développer culturellement la région. Ensuite, ces maisons doivent bénéficier d'appuis locaux qui les aident à publier des titres et qui participent à faire accroître leur visibilité régionale. De plus, un éditeur qui se dit «régional» doit nécessairement publier des titres qui traitent de l'histoire, de la littérature et de la culture de la région et, autant que possible, faire appel à des auteurs liés d'une façon ou d'une autre à cette même région, ce qui constitue le dernier critère définissant un éditeur régional.

Par l'historique, le fonctionnement et par la politique éditoriale de Mille Roches, nous vérifierons, dans les pages suivantes, la présence de ces quatre critères ainsi que la façon dont ils se manifestent. Nous prendrons alors connaissance de la philosophie de l'éditeur par rapport à sa région.

\section{Historique des Éditions Mille Roches}

Né en 1937 dans le Bas-du-Fleuve, Jean-Yves Théberge s'installa dans la région du Haut-Richelieu en 1952. Peu attiré par l'histoire de son village d'origine (Saint-Mathieu de Rimouski), Jean-Yves Théberge découvrit cependant que dans le Haut-Richelieu, il y avait abondance de sites et de bâtiments historiques. Il se mit donc à parcourir les routes, à lire les plaques sur les monuments, à visiter les églises et les cimetières du Haut-Richelieu. Plus il visitait cette région, plus il se documentait sur son histoire. Ses recherches l'ont amené à rencontrer d'autres personnes qui s'intéressaient à l'histoire régionale. Ces historiens écrivaient même, comme lui, sur ce qu'ils découvraient dans leurs recherches. La plupart des personnes rencontrées désiraient publier des livres sur leurs recherches et leurs découvertes. 
Mais à cette époque, la région de Saint-Jean comptait seulement quelques maisons d'édition qui publiaient par ailleurs peu de livres sur l'histoire du Haut-Richelieu, un créneau qui restait à développer dans cette région. Un besoin régional important n'était donc pas comblé et des pans d'histoire du Haut-Richelieu demeuraient ignorés de sa population.

Jean-Yves Théberge ne tenta pas de combler ce besoin à lui seul. Il sut s'entourer de partenaires qui contribuèrent à faire fonctionner et progresser les Éditions Mille Roches. En 1976, les historiens que Théberge avait rencontrés en menant ses recherches se réunirent pour discuter de la création d'une coopérative d'édition. Marie Gruslin fut invitée à cette réunion pour son expertise en gestion et en comptabilité. Elle informa le groupe du fonctionnement d'une coopérative, des processus décisionnels, etc. Afin d'éviter la discorde entre les écrivains qui seraient appelés à faire partie de la coopérative d'édition, Jean-Yves Théberge laissa tomber l'idée de la coopérative et décida de fonder, avec Marcel Colin et Marie Gruslin, une véritable maison d'édition, une compagnie au sens légal du terme. Théberge devint donc éditeur en partie par hasard, en partie par nécessité; c'est en somme sa curiosité pour l'histoire du HautRichelieu qui fut à l'origine des Éditions Mille Roches.

Nommé président de la maison dès sa fondation, JeanYves Théberge fit d'abord la connaissance de Marcel Colin aux Éditions du Richelieu et au Canada-français ${ }^{4}$. Au cours des ans, ils agiront tous deux à la fois comme directeurs littéraires et comme lecteurs des Éditions Mille Roches, prenant toujours les décisions en collégialité après avoir lu et discuté des manuscrits. C'est aussi une carrière dans l'enseignement qui a permis à Marie Gruslin et à Jean-Yves Théberge de se rencontrer, plusieurs années avant la fonda- 
tion de Mille Roches. Tant qu'elle fut associée aux Éditions Mille Roches, Marie Gruslin a agi à titre de comptable et s'est occupée des finances de la maison. Marcel Colin connaissait également Marie Gruslin avant la fondation de Mille Roches puisqu'il avait déjà enseigné avec la sœur de celle-ci.

C'est ainsi que Marcel Colin et Marie Gruslin se sont associés à Jean-Yves Théberge, en 1976, pour former le noyau fondateur des Éditions Mille Roches. Un trio de complices venait de se former et allait donner naissance à un bel exemple de maison d'édition régionale. Toutes les décisions, qu'elles soient d'ordre financier, publicitaire et parfois même littéraire, étaient prises à trois. Une fois la maison légalement mise sur pied, les trois fondateurs étaient prêts à entreprendre l'aventure de l'édition.

Ils baptisent la nouvelle institution du nom de «Mille Roches", qui renvoie à une anecdote historique. À l'époque de la Nouvelle-France, la navigation était un moyen de transport essentiel. Les voyageurs et les Amérindiens remontaient la rivière Richelieu avec leurs canots faits d'écorce. Comme l'eau était basse à l'automne, de nombreuses roches se retrouvaient à découvert dans la rivière. Les voyageurs s'avertissaient entre eux : «Faites attention! $\grave{A}$ tel endroit, il y a mille roches et vous allez briser vos canots 5 ». «Mille Roches» est également le nom d'origine d'Iberville, la ville voisine de Saint-Jean-sur-Richelieu. Comme la maison voulait faire connaître sa région, son histoire et sa culture, Jean-Yves Théberge se devait de choisir un nom doté d'une connotation symbolique et historique propre au Haut-Richelieu.

Le premier titre des Éditions Mille Roches fut lancé à l'automne 1976. L'essai historique du notaire Lionel Fortin ${ }^{6}$ fut le premier et le seul titre publié cette année-là. En 
l'honneur de la première publication de l'éditeur régional, Richard Lafontaine a écrit un long article dans Le Canadafrançais $^{7}$ qui présente les fondateurs, l'objectif de la maison, les prochaines publications, etc. En 1977, Mille Roches atteint sa vitesse de croisière. Quatre titres sont publiés : deux livres d'histoire régionale, un livre touristique et un autre d'artisanat. Au fil des ans, la réputation des Éditions Mille Roches a grandi grâce à des auteurs de la région qui ont contribué, par leurs œuvres, à faire connâtre la maison. Les essais historiques de Réal Fortin et de Pierre-B. Cadieux, les essais sur la vieillesse de Marcel Colin de même que le livre de Manon Leclair sur le tissage se sont très bien vendus, selon Jean-Yves Théberge. En quatorze ans d'existence, la maison a publié cinquante et un titres dont plus du tiers $(35 \%)$ sont des livres d'histoire régionale.

Mis à part les lancements, toujours agréables et chaleureux, la maison a connu, en 1985, un moment important de son histoire. Pour célébrer son neuvième anniversaire, Mille Roches a organisé une neuvaine littéraire «consacré[e] aux livres d'ici, aux auteurs d'ici, bref, à la culture d'ici ${ }^{8}$ \%. Plusieurs activités ont été tenues pendant ces neuf jours et l'événement a été un succès puisque pendant cette neuvaine littéraire, quelques centaines de personnes ont découvert un éditeur de leur région, un éditeur qu'ils ne connaissaient pas ou peu après une décennie d'existence à Saint-Jean-sur-Richelieu. Le président-fondateur était plutôt fier de cette initiative : «Nous voulions faire cette neuvaine pour mettre en vedette les auteurs d'ici et leurs volumes et je pense qu'on a bien réussi ${ }^{9} . »$

Le début des années quatre-vingts amène des changements au sein de l'équipe de direction des Éditions Mille Roches. Chroniqueur culturel au Canada-français, Richard 
Lafontaine se joint, en 1980, à l'équipe de Mille Roches. Quelques mois après l'arrivée de Lafontaine, Marie Gruslin quitte l'équipe de direction, car l'objectif que Jean-Yves Théberge lui avait fixé lors de la fondation (assurer une assise financière solide à la maison) était atteint. La maison ne retirait pas vraiment de profits, mais elle fonctionnait relativement bien.

Trois ans plus tard, en 1983 ou $1984^{10}$, Marcel Colin quitta à son tour les Éditions Mille Roches pour des raisons de santé et non par manque d'intérêt. Du trio, seuls Richard Lafontaine et Jean-Yves Théberge s'occupaient maintenant de faire fonctionner la maison. En 1985, Lafontaine décéda d'un cancer et Théberge resta seul à la barre de Mille Roches. Il assuma donc la direction de Mille Roches et toutes les tâches relatives à la maison pendant près d'un an et demi, jusqu'à ce qu'il prit la décision de la vendre aux frères Dulude. Ces derniers étaient propriétaires d'une librairie à Saint-Jean. Jean-Yves Théberge les connaissait déjà depuis un certain nombre d'années puisque Mille Roches participait volontiers aux événements littéraires que les Dulude organisaient dans leur librairie. Les frères Dulude étaient très intéressés à faire l'acquisition des Éditions Mille Roches. Après que les deux parties se furent entendues sur le montant de la transaction, l'acte de vente fut signé en mai 1986.

Pendant les années de direction des frères Dulude, la production de Mille Roches chuta : cinq titres en 1987, trois titres en 1988 et deux titres en 1989. Une mauvaise gestion financière et des décisions littéraires douteuses entrainèrent, dès 1989, la fin des activités d'édition de Mille Roches.

Une rupture très nette est évidente entre les deux périodes, soit celle pendant laquelle Théberge était le président de Mille Roches (1976-1986) et celle pendant laquelle 
les frères Dulude ont pris la "relève» (1986-1989). Marcel Colin résume bien cette rupture dans la politique éditoriale, ce changement d'orientation qui entraîna la disparition de Mille Roches :

La maison a été fondée pour la région et eux la voyait internationale... [...] C'était une affaire culturelle et tout d'un coup, c'est devenu une affaire commerciale.[...] Les Dulude [...] ont fait [de cette maison] une affaire d'argent alors que c'était, depuis le début, une affaire de cœur ${ }^{11}$.

Nées avant tout pour combler un besoin régional, les Éditions Mille Roches ont servi la population du Haut-Richelieu pendant près de quatorze ans. La disparition de la maison n'a depuis donné lieu à aucune autre initiative éditoriale du genre dans la région : depuis plus d'une décennie, les intérêts littéraires et culturels d'édition et de diffusion ne sont servis par aucun éditeur du Haut-Richelieu.

\section{Politique éditoriale et vocation régionale}

À travers la politique éditoriale de la maison, quelques aspects de son fonctionnement, les appuis régionaux, les titres publiés et les auteurs, nous voulons exposer la philosophie de l'éditeur en cernant les idéaux qu'il a véhiculés tout au long de son existence.

\section{a) La politique éditoriale}

Les choix éditoriaux de Mille Roches ont toujours été motivés par la mission régionale de ses fondateurs. Explicitement ou implicitement, la politique éditoriale visait surtout l'objectif suivant :

Ce que l'on voulait faire, c'était publier des ouvrages sur la région par des auteurs de la région autant que possible. C'était le principal but de la maison. 
[Notre politique éditoriale] était ouverte à tous les genres, à tout ce qui pouvait se présenter d'intéressant ${ }^{12}$.

La maison se définit elle-même comme une maison d'édition régionale : son objectif explicite était de découvrir et de faire connaitre les auteurs, l'histoire, la culture et le patrimoine de la région. Mille Roches se donne un rôle, une mission qui vise la découverte et la diffusion de la littérature et de la culture du Haut-Richelieu. Une autre facette de la politique éditoriale consistait à éveiller la conscience régionale. Comme le mentionne Jacques Boulerice ${ }^{13}$, il s'agissait de

donner à la Montérégie, à la région de Saint-Jean plus particulièrement, une vie littéraire qui n'était pas publiée avant la fondation de Mille Roches. Cette région a un passé extrêmement riche historiquement, une vie extrêmement riche littérairement parlant aussi. [...] C'était vraiment la politique éditoriale de Mille Roches : l'histoire et la vie littéraire de la région par les gens de la région. Le dénominateur commun était vraiment que ça appartienne à nos racines régionale ${ }^{14}$.

\section{b) Le fonctionnement}

En général, le fonctionnement de Mille Roches pourrait être qualifié d'artisanal : aucun employé n'a été engagé au fil des ans pour faire fonctionner l'éditeur, l'équipe de fondateurs se chargeait de toutes les étapes de la publication, de la soumission des manuscrits jusqu'à sa sortie en librairie. Pour démarrer Mille Roches, les fondateurs ont investi leur argent personnel. Jamais il n'a été question de retirer un quelconque profit avec la maison puisque le but 
premier était de faire découvrir les richesses culturelles de la région.

Le contact personnel était privilégié entre l'éditeur et les auteurs lorsque venait le temps de soumettre des manuscrits. Très souvent, les auteurs de la région allaient directement chez l'éditeur soumettre leur manuscrit en mains propres ${ }^{15}$. Ils avaient alors la chance de rencontrer JeanYves Théberge et de parler de leur manuscrit avant que le comité de lecture ne l'examine. À quelques reprises, Mille Roches a demandé à des auteurs du Haut-Richelieu de lui soumettre des manuscrits. En effet, à la suite de rencontres que Théberge tenait avec des auteurs, des projets d'écriture naissaient. C'est par exemple ce qui s'est produit dans le cas de Petite bistoire de Saint-Luc ${ }^{16}$ de Réal Fortin. Comme Mille Roches avait déjà fait paraitre un livre sur le village de L'Acadie ${ }^{17}$, Théberge considérait que publier l'histoire de Saint-Luc serait une bonne idée. Il en a donc parlé avec Réal Fortin qui, d'abord hésitant, a fini par accepter. JeanYves Théberge a aussi confié à Jacques Boulerice, dans les premières années de la maison, "que ça lui ferait bien plaisir $\mathrm{s}^{\prime}\left[i \mathrm{il]}\right.$ lui soumettai[t] des textes ${ }^{18} \%$. De cette proposition indirecte à Boulerice est né La boîte à bois ${ }^{19}$. Le comité de lecture, formé la plupart du temps par les fondateurs, faisait parfois appel à des professeurs ou des auteurs de SaintJean-sur-Richelieu, question d'avoir un avis extérieur. En ce qui concerne l'impression des livres, elle se faisait dans la grande région montérégienne. Mille Roches a longtemps octroyé les contrats à l'imprimerie Payette et Simms, à SaintLambert et, plus près d'eux, à l'imprimerie du Richelieu, à Saint-Jean-sur-Richelieu.

Un dernier aspect du fonctionnement de l'éditeur qui témoignait de ce souci de se rapprocher du milieu, de la population, est le lancement des titres de la maison. Les 
Éditions Mille Roches ont organisé des lancements dans plusieurs lieux du Haut-Richelieu. Lorsque le sujet du livre s'y prêtait, le lancement se déroulait dans un lieu en rapport avec ce livre. Les lancements des monographies relatant l'histoire d'une municipalité de la région se tenaient souvent à l'hôtel de ville de cette municipalité ou dans un lieu choisi pour sa valeur historique. Par exemple, le lancement du Couvent de Saint-Jean, 1847-198120 (Madeleine Héroux, 1984) s'est déroulé dans l'édifice du couvent et a donné lieu aux retrouvailles de plusieurs sœurs enseignantes. Pour le livre de Pierre-B. Cadieux, Le Fort Lennox ${ }^{21}$, le lancement a eu lieu au Fort Lennox même, à Saint-Paul-del'île-aux-Noix. Par ailleurs, la bibliothèque Adélard-Berger ${ }^{22}$ a été utilisée à plusieurs reprises pour des lancements de Mille Roches.

\section{c) Les collaborateurs régionaux}

Constituant un volet essentiel du fonctionnement de l'édition régionale, la collaboration d'acteurs locaux est très importante puisqu'elle soutient le travail de l'éditeur dans son milieu. Elle permet également à un éditeur régional d'être un acteur dynamique dans sa communauté en faisant participer plusieurs individus, groupes ou institutions locales à son activité éditoriale. Cette collaboration régionale a permis de mettre à contribution plusieurs ressources du Haut-Richelieu afin que les Éditions Mille Roches publient et se fassent connaître dans toute la région.

Les périodiques du Haut-Richelieu

Un éditeur aurait beaucoup de mal à se faire connâ̂tre dans sa région sans l'aide des hebdomadaires locaux. Achetés et lus par des milliers de personnes chaque semaine, les hebdos sont un appui de taille pour un éditeur régional 
désireux d'annoncer ses publications et ses activités à la communauté. Pour les Éditions Mille Roches, quatre périodiques ont joué ce rôle à divers degrés. Tout d'abord, mentionnons trois hebdomadaires locaux ${ }^{23}$ qui ont, à tour de rôle, annoncé les Éditions Mille Roches, soit par des chroniques «vient de paraître», par des articles consacrés aux lancements de Mille Roches ou par des comptes rendus portant sur les livres de l'éditeur. Ces hebdos, souvent distribués gratuitement à la population du Haut-Richelieu, contiennent beaucoup de publicités et d'articles relatant divers événements politiques, économiques et culturels qui surviennent dans la région.

Considéré comme la voix de diffusion majeure dans le Haut-Richelieu depuis sa fondation en $1860^{24}$, Le Canadafrançais a rapporté la grande majorité des lancements et des activités de Mille Roches en plus de publier des comptes rendus de presque tous les livres de la maison. Le Canadafrançais a joué un rôle capital pour l'éditeur régional, comme nous l'a confié Jean-Yves Théberge :

Le journal était très généreux pour nous! Il nous faisait de la publicité gratuite puisqu'il parlait beaucoup de nos livres lors de leur parution. Michel Phaneuf et Jean-François Crépeau ont fait de bonnes critiques de nos livres. Michel Phaneuf nous aidait beaucoup parce qu'il mettait des photos du lancement dans le journal et qu'il parlait beaucoup du lancement. Ça nous aidait à faire vendre nos livres, c'est certain. [...] Le journal a toujours été un bon alliée 25 .

L'importance de la mission régionale a toujours uni les Éditions Mille Roches et Le Canada-français. Ce sens de la mission régionale a même sauvé le sort de certains livres. À propos du roman Le cour absent ${ }^{26}$, de Gilles Gemme, 
Pierre Dulude (l'un des nouveaux propriétaires de Mille Roches) explique par exemple que :

M. Crépeau nous faisait des bonnes critiques même si, par exemple, l'ouvrage de Gilles Gemme n'était pas réussi. Il aurait pu facilement nous descendre dans sa critique, mais il ne l'a pas fait. En région, même si certaines choses sont de mauvaise qualité, on ne les descend pas pour protéger notre région en quelque sorte. Il faut se tenir et c'est ce que M. Crépeau a fait $^{27}$.

Le Canada-français et ses chroniqueurs littéraires ont contribué à créer et à maintenir une vie littéraire et culturelle dans le Haut-Richelieu en unissant leurs efforts à ceux des animateurs de Mille Roches. Chacun a su promouvoir, à sa façon, l'histoire, la littérature et la culture de la région auprès des citoyens et des autorités locales.

\section{Les institutions locales}

Nous savons maintenant que les périodiques du HautRichelieu se sont fait un point d'honneur de faire connầtre Mille Roches et d'inciter les lecteurs à se procurer les livres de l'éditeur. En ce qui concerne les librairies et les bibliothèques de la région, c'était presque une obligation morale de mettre en vitrine les livres de Mille Roches ou de les conserver dans leurs rayons (bibliothèques municipales, bibliothèques des écoles primaires et secondaires, bibliothèque du Cégep Saint-Jean-sur-Richelieu).

La collaboration des libraires est indispensable : ceuxci présentent aux citoyens de la région les livres qui les concernent au premier chef. Jean-Yves Théberge se souvient que la Librairie du Richelieu ainsi que la librairie des frères Dulude les ont toujours soutenus en commandant des li- 
vres de Mille Roches et en les mettant en évidence sur leurs rayons et dans leurs vitrines.

En ce qui concerne les institutions scolaires et les bibliothèques municipales, leurs responsables n'ont jamais manqué d'acheter les titres de Mille Roches. Selon les clientèles qu'ils desservent, les établissements d'enseignement ainsi que les bibliothèques municipales ont fait l'acquisition de la plupart des titres du catalogue de Mille Roches. Par exemple, la bibliothèque municipale Adélard-Berger s'est procuré $80 \%$ des titres du catalogue qui en contient cinquante et un, et la bibliothèque du Cégep de Saint-Jean en a acquis $55 \%$. "On avait une bonne collaboration de la région ${ }^{28} »$, conclut Pierre Dulude.

\section{Les artistes}

Par sentiment de fierté envers leur propre région, les directeurs de Mille Roches ont fait appel à des artistes locaux (peintres, dessinateurs, illustrateurs, graphistes) pour illustrer la page couverture et l'intérieur des livres. "C'était [...] pour encourager les artistes de la région ${ }^{29} »$, confirme Jean-Yves Théberge. Au total, dix-huit artistes ont collaboré à une vingtaine de publications de Mille Roches par des toiles, des aquarelles, des dessins ou des illustrations. Nous savons qu'au moins seize des dix-huit artistes qui ont collaboré avec Mille Roches provenaient du Haut-Richelieu. Par exemple, dans le cas de Présence urbaine ${ }^{30}$ d'Yvan Lafontaine, deux artistes qui jouissent d'une très grande visibilité dans la région, Réal Dumais et Pierre Ouvrard, ont respectivement réalisé la typographie et l'emboîtage du livre d'artiste.

Parfois, c'est l'éditeur qui a amené un artiste à collaborer avec un auteur, mais il est également arrivé que des auteurs ont proposé à Mille Roches un artiste régional pour 
illustrer leur livre. «C'était la plupart du temps les auteurs qui nous amenaient un illustrateur qu'ils connaissaient et qu'ils voulaient pour travailler sur leur ouvrage. Il y a eu Daniel Laverdure, Louise Filion, Michèle Côté, MarieAndrée Lestage, entre autres ${ }^{31} »$, se souvient Pierre Dulude.

Plusieurs de ces artistes ont collaboré bénévolement avec des auteurs tandis que d'autres ont reçu un montant d'argent, symbolique la plupart du temps. Cependant, dans le cas du roman de Lise Bourdeau ${ }^{32}$, Mille Roches a fait appel à un peintre reconnu dans le Haut-Richelieu, Roger Alexandre, en lui commandant une toile pour la première de couverture de ce roman. Mille Roches a déboursé quatre cents dollars ${ }^{33}$ pour cette peinture et Jean-Yves Théberge a par la suite conservé la toile.

Les photographes

Une vingtaine de titres des Éditions Mille Roches contiennent des photographies de l'auteur du livre (en quatrième de couverture) et/ou du sujet. Ces photos ont été prises par une douzaine de photographes, professionnels ou amateurs, dont Jacques Paul, photographe attitré du Canada-français depuis de nombreuses années ${ }^{34}$. À lui seul, Jacques Paul a collaboré à huit livres entre 1977 et 1989 . Sur les douze photographes qui ont collaboré à la présentation matérielle des livres de Mille Roches, dix étaient domiciliés dans le Haut-Richelieu à l'époque, l'un provenait de Saint-Hilaire et l'autre de l'extérieur de la Montérégie. Comme nous l'avons vu dans le cas des artistes, certains photographes ont été rémunérés alors que d'autres ont offert gratuitement leurs services. Quelques auteurs, dont Réal Fortin et Léo Tremblay, ont pris eux-mêmes des photos pour certains de leurs livres. 
La conception graphique

Jean-Yves Théberge a rencontré Nicole Houle lorsque tous deux travaillaient au Canada-français, lui comme chroniqueur littéraire et elle comme graphiste. Richard Lafontaine ${ }^{35}$ les a présentés l'un à l'autre, et cela a marqué le début d'une longue collaboration professionnelle. Nicole Houle a réalisé la conception graphique et le montage d'au moins $70 \%{ }^{36}$ des livres de Mille Roches. Elle a commencé à collaborer avec l'éditeur de Saint-Jean dès 1978 et est restée fidèle à la maison jusqu'en 1989, réalisant même la conception graphique et le montage des deux derniers titres de Mille Roches. Roch Tanguay, lui-même un auteur de Mille Roches, a réalisé la conception graphique et le montage de cinq livres de la maison en plus d'avoir fait des dessins pour deux autres titres.

\section{Les appuis financiers}

Dans le cas de certains titres, les Éditions Mille Roches ont pu compter sur un appui financier provenant de la région. Pour Petite histoire de Saint-Luc, les Éditions Mille Roches ont bénéficié d'un appui financier de la part de la ville de Saint-Luc : «[...] la municipalité nous a acheté un certain nombre d'exemplaires à un bon prix, à un prix supérieur au prix de vente en librairie. Autrement dit, elle nous donnait une subvention ${ }^{37}$ \%. Par ailleurs, l'éditeur régional a également reçu une contribution financière des municipalités concernées par les ouvrages Nelson Mott et l'bistoire de Saint-Jean et Histoire de L'Acadie du Haut-Richelieu $^{38}$. 
d) Titres des livres et contenu régional

Toujours dans l'optique de la politique éditoriale de Mille Roches qui privilégie la publication de titres qui portent sur la région par des auteurs de la région, nous avons fait le décompte des livres affichant un titre qui réfère au Haut-Richelieu. D'abord, la totalité des dix-huit livres d'histoire régionale recensés dans le catalogue de l'éditeur ont un titre qui est relié explicitement à la région, soit par une personnalité importante qui y a vécu (Nelson Mott et l'histoire de Saint-Jean, par exemple ${ }^{39}$ ), soit par un bâtiment significatif de l'histoire locale (Le Fort Lennox ${ }^{40}$ ) ou par un rappel de l'histoire d'une municipalité du Haut-Richelieu (Petite histoire de Saint-Luc ${ }^{41}$, entre autres). De même, un titre peut rappeler une réalité historique de la région (Croisière sur le Richelieu bistorique ${ }^{42}$ ), un événement marquant survenu dans cette même région (L'avènement du premier chemin de fer au Canada: Saint-Jean/Laprairie, 183643), etc. Grâce à ces dix-huit titres d'histoire régionale, $35 \%$ de la production de Mille Roches a un lien explicite avec l'histoire du Haut-Richelieu.

Outre les livres qui portent explicitement sur l'histoire de la région, d'autres publications ont un titre qui évoque, plus ou moins explicitement, une personne ou un événement du Haut-Richelieu. Parmi celles-ci, nous pouvons mentionner trois biographies (Félix-Gabriel Marchand $^{44}$; Georges E. Tremblay, sculpteur, 1878-193945; et La vie beureuse de Léopold $T$., camionneur $\left.{ }^{46}\right)$, trois titres consacrés au tourisme régional (Les constructions militaires $d u$ Haut-Richelieu. Guide touristique ${ }^{47}, \ldots A$ pied dans le vieux Saint-Jean ${ }^{48}$, Cap sur le Richelieu ${ }^{49}$ ) et trois livres jeunesse (Le Haut-Richelieu : album de jeu pour les 7 à 12 ans $5^{50}$, Le gros chat de fer et de feu ${ }^{51}$ et Si j'étais une montgolfière ${ }^{52}$ ). À ces titres qui évoquent la région s'ajoutent un roman ${ }^{53}$, une 
pièce de théâtre $\mathrm{e}^{54}$, un manuel scolaire ${ }^{55}$ et un livre sur la poterie de Saint-Jean et d'Iberville.

Le contenu de tous ces livres, qui appartiennent à divers genres et catégories littéraires, est lié de différentes façons à la région. Au total, si l'on inclut les dix-huit livres d'histoire régionale, trente et un livres de Mille Roches (sur les cinquante et un qui composent le catalogue) ont un titre et/ou un contenu qui se rapportent, en tout ou en partie, à la région du Haut-Richelieu. En somme, près de $61 \%$ du catalogue des Éditions Mille Roches est composé de titres qui se rapportent plus ou moins explicitement à la région.

\section{e) Les auteurs}

Tout d'abord, même si nous n'avons pu retracer le lieu d'origine de tous les auteurs à l'aide des dictionnaires consultés, Jean-Yves Théberge nous a confirmé que les vingthuit auteurs qui ont publié chez Mille Roches habitaient le Haut-Richelieu ou la grande région montérégienne à l'époque de la publication de leur(s) livre(s). Nous avons pu recueillir des informations substantielles sur onze des vingthuit auteurs qui ont publié aux Éditions Mille Roches. Le profil socio-professionnel de ces onze auteurs met en relief les liens qui les reliaient ou les relient encore avec le HautRichelieu.

Sept auteurs sur les onze étudiés sont nés dans le HautRichelieu ou dans la grande région de la Montérégie, tous, sauf deux exceptions, ont enseigné ou enseignent encore dans le Haut-Richelieu. En ce qui concerne les types de publications et les éditeurs de ces auteurs, nous avons noté que la plupart (sept sur onze) ont publié au moins un livre qui affiche un contenu régional lié au Haut-Richelieu, que ce contenu soit historique, culturel, touristique, généalogique ou autre. De plus, comme il s'agit d'auteurs des Édi- 
tions Mille Roches, les onze ont bien sûr publié au moins un titre chez un éditeur régional. Mais plusieurs d'entre eux ont également publié chez d'autres éditeurs du HautRichelieu avant, pendant ou après la période d'existence de Mille Roches. Enfin, cinq auteurs de Mille Roches ont collaboré au Canada-français, que ce soit à titre de chroniqueur régulier ou d'auteur occasionnel de comptes rendus, et cinq autres ont entretenu des rapports professionnels avec des périodiques ou des organismes culturels du Haut-Richelieu, comme le Musée régional et la Corporation des musées de Saint-Jean. L'analyse de ces éléments nous fait prendre conscience du sentiment d'appartenance régionale de plusieurs auteurs. Que se soit parce qu'ils sont nés dans le Haut-Richelieu ou bien parce que leurs activités professionnelles (enseignement, édition, collaboration au Canada-français, etc.) y étaient situées, le profil socio-professionnel de ces onze auteurs confirme leur sentiment d'appartenance à la région johannaise.

\section{$* * *$}

Dans le cas des Éditions Mille Roches, la distinction entre «éditeur en région» et "éditeur régional» mérite d'être soulignée à nouveau puisqu'elle prend toute son importance dans le cadre de cette étude. Cet éditeur ne s'est pas seulement contenté d'élire domicile en dehors de la métropole (comme le ferait un éditeur en région) et de ne pas tenir compte des richesses de la région où il s'installe. Mille Roches, en tant qu'éditeur régional, voulait précisément combler des lacunes historiques, culturelles et littéraires dans le Haut-Richelieu. De plus, il voulait faire connaître la région et ses richesses et, indirectement, il désirait faire naître une conscience régionale en impliquant les auteurs, les institutions, les artistes, la population, etc., à toutes les étapes de 
l'activité éditoriale. Par le biais de cette conscientisation s'est développé un sentiment d'appartenance à la région partagé par tous les acteurs ayant œuvré, de près ou de loin, avec les Éditions Mille Roches.

Pour nous permettre de bien cibler ce qu'est un éditeur régional, nous avons élaboré quatre critères le définissant et ils méritent qu'on y revienne puisqu'ils constituent le fondement de cette étude. Tout d'abord, les Éditions Mille Roches ont été fondées par une équipe et non par une seule personne. Cela représente l'une des forces de la maison: mettre à profit le talent, le dévouement et l'expertise de Jean-Yves Théberge, Marie Gruslin et Marcel Colin pour assurer des assises solides à la maison d'édition. Ensuite, les nombreux appuis locaux dont a bénéficié Mille Roches l'ont aidée à se faire connaître dans le Haut-Richelieu et à survivre par l'implication, la plupart du temps bénévole, d'artistes, d'institutions locales, de périodiques (Le Canada-francais), de photographes, de concepteurs graphiques et d'imprimeurs (les seuls qui ont toujours été rémunérés). Sans ces appuis, la vocation régionale de la maison aurait sans doute été moins marquée.

Comme Mille Roches voulait faire connaitre l'histoire de sa région, l'éditeur a donné la priorité aux ouvrages traitant de personnes, de monuments, d'institutions et d'événements du Haut-Richelieu. Le catalogue de l'éditeur, composé à 35\% de titres d'histoire régionale, illustre bien cette mission régionale que se sont donnée les fondateurs de Mille Roches et l'importance de faire sortir de l'ombre les richesses historiques. Enfin, il ne manquait que l'apport d'auteurs locaux pour compléter la vocation régionale de l'éditeur. Tous les auteurs ayant collaboré avec Mille Roches habitaient ou provenaient du Haut-Richelieu et ont accepté de s'impliquer dans la poursuite de la mission ré- 
gionale de l'éditeur parce qu'il était important pour eux de contribuer à l'essor culturel, historique et littéraire de leur région. Par le fait même, leur sentiment d'appartenance au Haut-Richelieu s'est renforcé.

Avec ses trois fondateurs, ses divers appuis locaux qui l'ont aidée et soutenue, ses nombreux titres sur l'histoire régionale rédigés par des auteurs locaux, Mille Roches a sans aucun doute tenu un rôle prépondérant dans le HautRichelieu, a rempli une mission régionale pendant les treize années de son existence. Cette réussite locale est due au dévouement de toutes les personnes (fondateurs, auteurs, collaborateurs régionaux) qui se sont fait un devoir de faire connaître cette région au passé historique extrêmement riche, et surtout, qui ont cru possible d'intéresser la population qui méritait de découvrir ces pans d'histoire régionale.

Dans l'aventure des Éditions Mille Roches, nous devons avant tout retenir que ce n'était pas seulement les fondateurs, mais également les auteurs, qui participaient à la mission régionale de l'éditeur, puisqu'ils se sentaient tout aussi concernés que les directeurs de la maison. En fait, tous les acteurs culturels importants du Haut-Richelieu, que ce soit des individus, des organismes ou des institutions, ont mis la main à la pâte pour faire des Éditions Mille Roches un succès régional. Cependant, depuis sa disparition en 1989, aucun autre éditeur du Haut-Richelieu n'a voulu entretenir et poursuivre cette mission régionale ou n'a su mobiliser en ce sens les ressources de la région. Cela montre, d'une part, la difficulté de fonder une entreprise culturelle à vocation régionale, et, d'autre part, que personne n'a depuis voulu investir les efforts nécessaires pour développer ce type de maison d'édition : très peu empruntent la voie de la culture en passant volontairement à côté du chemin du profit... 


\section{NOTES}

${ }^{1}$ Titulaire d'une maîtrise en études françaises de l'Université de Sherbrooke.

${ }^{2}$ Fernand Harvey, «L'édition régionale au Québec : entrepreneurship, affirmation culturelle et reconnaissance institutionnelle», dans Fernand Harvey et Andrée Fortin, dir. La nouvelle culture régionale, Québec, Institut québécois de recherche sur la culture (IQRC), 1995, p. 191192.

${ }^{3}$ Nous définirons plus loin ce concept.

${ }^{4}$ Le Canada-franfais est l'hebdomadaire du Haut-Richelieu.

${ }^{5}$ Entrevue de Caroline Béland avec Jean-Yves Théberge, L'Acadie, 3 juin 1999, document inédit (1 heure et 15 minutes, 16 feuillets), f. 2.

${ }^{6}$ Lionel Fortin, Nelson Mott et l'bistoire de Saint-Jean, Saint-Jean-surRichelieu, Éditions Mille Roches, 1976, 109 p.

7 Richard Lafontaine, «Les Éditions Mille Roches : pour mieux faire connaître la région et nos écrivains», Le Canada-français, vol. $117, \mathrm{n}^{\circ}$ 23 (3 novembre 1976), p. 30.

${ }^{8}$ Michel Phaneuf, «La neuvaine de Mille Roches : neuf jours de fête littéraire à la bibliothèque», Le Canada-français, vol. 126, $\mathrm{n}^{\circ} 15$ (11 septembre 1985), p. A-48.

${ }^{9}$ Michel Phaneuf, «Neuf jours qui ont fait mieux connaitre les livres et les auteurs de la région», Le Canada-français, vol. 126, $\mathrm{n}^{\circ} 17$ (25 septembre 1985), p. A-52.

${ }^{10} \mathrm{La}$ date du départ de Marcel Colin est imprécise parce que personne, parmi ceux qui furent interviewés, n'a pu s'en rappeler avec certitude.

${ }^{11}$ Entrevue de Caroline Béland avec Marcel Colin, Montréal, 16 juin 1999, document inédit (1 heure, 8 feuillets), f. 5 et f. 7.

${ }^{12}$ Entrevue de Caroline Béland avec Jean-Yves Théberge, op. cit., f. 4-5.

${ }^{13}$ Jacques Boulerice, poète reconnu dans le Haut-Richelieu, a publié trois titres aux Éditions Mille Roches.

${ }^{14}$ Entrevue de Caroline Béland avec Jacques Boulerice, Montréal, 19 février 1999, document inédit (1 heure et 45 minutes, 14 feuillets), f. 7 . 
${ }^{15}$ En région, le cercle littéraire est beaucoup plus restreint que dans les grandes villes. Presque tous les auteurs et les éditeurs se connaissent, ce qui favorise le contact direct et personnel lorsque arrive le temps de déposer un manuscrit chez l'éditeur. Cette pratique est très peu fréquente à Montréal, le grand nombre de manuscrits reçus par un éditeur ne lui donnant pas le temps de rencontrer personnellement un auteur.

${ }^{16}$ Réal Fortin, Petite histoire de Saint-Luc, Saint-Jean-sur-Richelieu, Éditions Mille Roches, 1978, 159 p.

${ }^{17}$ Il s'agit de L'Acadie et son église (1977), de Pierre Brault.

${ }^{18}$ Entrevue de Caroline Béland avec Jacques Boulerice, op. cit., f. 7.

${ }^{19}$ Jacques Boulerice, La boîte à bois, Saint-Jean-sur-Richelieu, Éditions Mille Roches, 1978, 141 p.

${ }^{20}$ Madeleine Héroux, Le couvent de Saint-Jean, 1847-1981, Saint-Jeansur-Richelieu, Éditions Mille Roches, 1984, 141 p.

${ }^{21}$ Pierre-B. Cadieux, Le Fort Lennox, Saint-Jean-sur-Richelieu, Éditions Mille Roches, 1986, 119 p.

${ }^{22}$ Il s'agit de la bibliothèque de la ville de Saint-Jean-sur-Richelieu.

${ }^{23}$ Ces trois hebdomadaires n'en forment qu'un en réalité puisqu'il s'agit du même périodique qui, au fil des ans, a changé de nom. Il s'agit du Richelieu (1935-1978), du Richelieu agricole (1978-1987) et du Richelieu dimanche (1987- ).

${ }^{24}$ Le Canada-fransais a d'abord été connu sous le nom Le Franco-canadien. Celui-ci a été absorbé par Le Canada-franfais en 1895. Pendant soixante-dix ans, le journal conservera la double appellation $\mathrm{Le} C \mathrm{Ca}$ nada-français/Le Franco-canadien. Depuis plusieurs années, Le Canadafrançais est tiré à en moyenne dix-sept mille exemplaires hebdomadairement.

${ }^{25}$ Entrevue de Caroline Béland avec Jean-Yves Théberge, op. cit., f. 12.

${ }^{26}$ Gilles Gemme, Le cour absent, Saint-Jean-sur-Richelieu, Éditions Mille Roches, 1989, 151 p.

${ }^{27}$ Entrevue de Caroline Béland avec Pierre Dulude, Victoriaville, 13 septembre 1999, document inédit (35 minutes), f. 4.

${ }^{28}$ Ibid., f. 4. 
${ }^{29}$ Entrevue de Caroline Béland avec Jean-Yves Théberge, op. cit., f. 8.

${ }^{30}$ Yvan Lafontaine, Présence urbaine. Gravure en couleurs avec un texte de Jacques Boulerice et un texte de Jean-Yves Théberge, Saint-Jean-sur-Richelieu, Éditions Mille Roches, 1987, 3 f.

${ }^{31}$ Entrevue de Caroline Béland avec Pierre Dulude, op. cit., f. 3.

${ }^{32}$ Lise Bourdeau, Josée : récit d'un inceste, Saint-Jean-sur-Richelieu, Éditions Mille Roches, 1982, 214 p.

${ }^{33}$ Ce montant est de loin le plus important que Mille Roches ait payé à un artiste pour sa contribution à un livre.

${ }^{34}$ Jacques Paul est d'ailleurs toujours à l'emploi du Canada-francais.

${ }^{35}$ Richard Lafontaine s'occupait, dans les années soixante-dix, de la section «Arts et spectacles» du Canada-français et a par la suite joint l'équipe des fondateurs de Mille Roches, en 1980.

${ }^{36}$ Nous croyons que Nicole Houle a réalisé la conception graphique de près de $85 \%$ des livres de Mille Roches, mais comme plusieurs conceptions graphiques ne sont pas signées, nous ne pouvons l'affirmer avec certitude.

${ }^{37}$ Entrevue de Caroline Béland avec Jean-Yves Théberge, op. cit., f. 12.

${ }^{38}$ Pierre Brault, Histoire de L'Acadie du Haut-Richelieu, Saint-Jean-surRichelieu, Éditions Mille Roches, 1982, 316 p.

${ }^{39}$ Nelson Mott fut, de 1848 à 1850, le premier maire de Saint-Jean (Lionel Fortin, Nelson Mott et l'bistoire de Saint-Jean, op. cit.).

${ }^{40}$ Pierre-B. Cadieux, Le Fort Lennox, op. cit.

${ }^{41}$ Réal Fortin, Petite histoire de Saint-Luc, op. cit.

${ }^{42}$ Pierre-B. Cadieux, Croisière sur le Richelieu historique, Saint-Jean-surRichelieu, Éditions Mille Roches, 1977, 47 p.

${ }^{43}$ François Cinq-Mars, L'Avènement du premier chemin de fer au Canada: St-Jean/Laprairie, 1836, Saint-Jean-sur-Richelieu, Éditions Mille Roches, 1986, 223 p.

${ }^{44}$ Avant de devenir Premier ministre du Québec, Marchand était député dans le Haut-Richelieu depuis 1867 (Lionel Fortin, Félix-Gabriel Marchand, Saint-Jean-sur-Richelieu, Éditions Mille Roches, 1979, 232 p.). 
${ }^{45}$ Le sculpteur Georges E. Tremblay a passé sa vie à Saint-Jean-surRichelieu et ses œuvres ont été marquantes pour la région (Marcel Colin, Georges E. Tremblay, sculpteur, 1878-1939, Saint-Jean-sur-Richelieu, Éditions Mille Roches, 1985, 79 p.).

${ }^{46}$ L'auteur du livre a écrit cet ouvrage pour rectifier les faits à la suite du film réalisé par Gilles Carle, La vie beureuse de Léopold $Z$. Léo Tremblay possédait une entreprise de camionage à Saint-Jean-sur-Richelieu (Léo Tremblay, La vie beureuse de Léopold T., camionneur, SaintJean-sur-Richelieu, Éditions Mille Roches, 1988, 208 p.).

${ }^{47}$ Pierre-B. Cadieux et Réal Fortin, Les constructions militaires du HautRichelieu. Guide touristique, Saint-Jean-sur-Richelieu, Éditions Mille Roches, 1977, 123 p.

${ }^{48}$ Roch Tanguay et Jean-Yves Théberge, ... À pied dans le vieux SaintJean, Saint-Jean-sur-Richelieu, Éditions Mille Roches, 1978, 119 p.

${ }^{49}$ Nancy Baron et al., Cap sur le Richelieu, Saint-Jean-sur-Richelieu, Éditions Mille Roches, 1985, 87 p.

${ }^{50}$ Réal Fortin, Le Haut-Richelieu : album de jeux pour les 7 à 12 ans, Saint-Jean-sur-Richelieu, Éditions Mille Roches, 1979, 23 p.

${ }^{51}$ Le gros chat de fer et de feu fait allusion à une locomotive et le livre renvoie à la première ligne de chemin de fer au Canada, ouverte en 1836, qui liait Saint-Jean à Laprairie Jean-Yves Théberge, Le gros chat de fer et de feu, Saint-Jean-sur-Richelieu, Éditions Mille Roches, 1987, 28 p.).

${ }^{52}$ Ce livre jeunesse, qui traite des montgolfières, fait référence au Festival des montgolfières de Saint-Jean-sur-Richelieu qui se déroule chaque été depuis 1984 (Jean-Yves Théberge, Si j'étais une montgolfière, Saint-Jean-sur-Richelieu, Éditions Mille Roches, 1988, 31 p.).

${ }^{53}$ L'intrigue du roman Le diable à quatre, de Réal Fortin, se déroule sur le bord de la rivière Richelieu (Réal Fortin, Le diable à quatre, SaintJean-sur-Richelieu, Éditions Mille Roches, 1980, 171 p.).

${ }^{54}$ L'action de la pièce de théâtre de Richard Evraire se situe au Collège militaire de Saint-Jean-sur-Richelieu (Richard Evraire, Chambre 204, Saint-Jean-sur-Richelieu, Éditions Mille Roches, 1982, 86 p.).

${ }^{55}$ Le musée s'en vient à l'école est un petit manuel pédagogique conçu pour initier les élèves du Haut-Richelieu au Musée régional et pour les préparer à une éventuelle visite du musée (Réal Fortin et Élizabeth 
Labenski-Gervais, Le musée s'en vient à l'école, Saint-Jean-sur-Richelieu, Éditions Mille Roches, 1985, 24 p.). 